This document is published in:

Combustion and Flame, Vol. 160, no 10, pp. 1981-1989

DOI: http://dx.doi.org/10.1016/j.combustflame.2013.04.027

(C) 2013 The Combustion Institute. Published by Elsevier Inc. 


\title{
Hydrogen-air mixing-layer ignition at temperatures below crossover
}

\author{
Eduardo Fernández-Tarrazo a ${ }^{\text {, Antonio L. Sánchez }}{ }^{\mathrm{a}, \mathrm{b}, *}$, Forman A. Williams ${ }^{\mathrm{b}}$ \\ a Dept. Ingeniería Térmica y de Fluidos, Universidad Carlos III de Madrid, Leganés 28911, Spain \\ ${ }^{\mathrm{b}}$ Dept. Mechanical and Aerospace Engineering, University of California San Diego, La Jolla, CA 92093-0411, USA
}

Keywords:

Hydrogen ignition

Reduced chemistry

Induction time

Crossover temperature

Activation-energy asymptotics

\begin{abstract}
A B S T R A C T
This paper addresses ignition histories of diffusion flames in unstrained hydrogen-air mixing layers for initial conditions of temperature and pressure that place the system below the crossover temperature associated with the second explosion limit of hydrogen-oxygen mixtures. It is seen that a two-step reduced chemical-kinetic mechanism involving as main species $\mathrm{H}_{2}, \mathrm{O}_{2}, \mathrm{H}_{2} \mathrm{O}$, and $\mathrm{H}_{2} \mathrm{O}_{2}$, derived previously from a detailed mechanism by assuming all radicals to follow a steady-state approximation, suffices to describe accurately the ignition process. The strong temperature sensitivity of the corresponding overall rates enables activation-energy asymptotics to be employed for the analysis, following the ideas developed for mixing-layer ignition by Liñán and Crespo in 1976 on the basis of one-step Arrhenius model chemistry. When the initial temperatures of both reactants differ by a relative amount that is of the order of or smaller than the ratio of this temperature to the effective activation temperature, the chemical reaction is seen to occur at a significant rate all across the mixing layer. The ignition time is then determined as a thermal runaway in a parabolic problem describing the evolution of the temperature increment and the $\mathrm{H}_{2} \mathrm{O}_{2}$ concentration, with local accumulation, chemical reaction, and transverse convection and diffusion, all being important. By way of contrast, when the air side is sufficiently hotter than the hydrogen side, as often occurs in applications, ignition occurs in a thin layer close to the air-side boundary, enabling a simplified description to be developed in which the ignition time is determined by analyzing the existence of solutions to a two-point boundary-value problem involving quasi-steady diffusion-reaction ordinary differential equations.
\end{abstract}

\section{Introduction}

The underlying chemistry and the associated ignition history involved in the self-ignition of hydrogen-air diffusion flames are very different depending on the initial temperature and pressure. Thus, for conditions that place the system above the so-called crossover temperature, a pressure-dependent quantity that defines the second explosion limit of hydrogen-oxygen mixtures [1], the resulting ignition process takes place as a branched-chain explosion, a problem that has been studied at length because of its importance for applications such as supersonic combustion in SCRAMJET systems. On the other hand, a thermal explosion characterizes ignition below crossover, a result first derived rigorously by Treviño [2].

Hydrogen-air ignition below crossover is of general interest especially for safety issues associated with planning of a future hydrogen economy. Detailed knowledge of the ignition time is needed, for instance, for designing reliable mixing systems in hydrogen-fired (or syngas-fired) gas turbines. The conditions in the mixers in premixed gas turbines upstream from the combus-

* Corresponding author at: Dept. Mechanical and Aerospace Engineering, University of California San Diego, La Jolla, CA 92093-0411, USA. Fax: +34 916249430.

E-mail address: asanchez@ing.uc3m.es (A.L. Sánchez). tion chamber include pressures in the range 10-20 atm and temperatures of the air stream typically on the order of $700-800 \mathrm{~K}$, thereby placing the system below the second explosion limit. Increasing the compression ratio to higher values, as required to achieve higher turbine efficiencies, increases the air temperature, resulting in smaller autoignition times that may give rise to undesired flame formation in the mixer as the fuel is injected. Detailed knowledge of mixing-layer ignition times at temperatures below crossover is therefore needed in assessing safe operation of these devices.

As shown in [2,3], hydrogen ignition below crossover takes place as a thermal explosion, controlled by slow reactions involving hydroperoxyl and hydrogen peroxide, whereas the radicals $\mathrm{H}$, $\mathrm{O}$, and $\mathrm{OH}$, which dominate the branched-chain explosion that characterizes ignition above crossover [4], are consumed at a fast rate, with the result that these species follow closely a steadystate approximation. The most important elementary reactions are $2 \mathrm{HO}_{2} \stackrel{6}{\rightarrow} \mathrm{H}_{2} \mathrm{O}_{2}+\mathrm{O}_{2}, \quad \mathrm{HO}_{2}+\mathrm{H}_{2} \stackrel{7}{\rightarrow} \mathrm{H}_{2} \mathrm{O}_{2}+\mathrm{H}, \quad$ and $\mathrm{H}_{2} \mathrm{O}_{2}+$ $\mathrm{M} \stackrel{8}{\rightarrow} 2 \mathrm{OH}+\mathrm{M}$, the latter exhibiting significant falloff at pressures typical of gas-turbine operation. Uncertainties in reaction-rate parameters still exist for all three reactions, with the degree of uncertainly being particularly high in connection with reaction 7 , for which very few experimental measurements are available in 
the range of temperatures of interest. As for the hydrogen-peroxide dissociation reaction 8 , the main deficiencies in rate modeling pertain to chaperon efficiencies, with no specific recommendation currently available for $\mathrm{H}_{2}$, its value being inferred from other recombination reactions.

The chemistry describing the thermal explosion can be simplified, following a recent analysis of homogeneous ignition [3], by noting that, except during a short initial period, hydroperoxyl consumption by $2 \mathrm{HO}_{2} \stackrel{6}{\rightarrow} \mathrm{H}_{2} \mathrm{O}_{2}+\mathrm{O}_{2}$ is fast enough to place this radical in steady state. Introducing this approximation, along with accompanying steady-state assumptions for $\mathrm{H}, \mathrm{O}$, and $\mathrm{OH}$, is seen to reduce the chemistry to the two overall steps $2 \mathrm{H}_{2}+\mathrm{O}_{2} \rightarrow 2 \mathrm{H}_{2} \mathrm{O}$ and $\mathrm{H}_{2}+\mathrm{O}_{2} \rightarrow \mathrm{H}_{2} \mathrm{O}_{2}$, with rates that exhibit a strong temperature sensitivity [3].

Diffusion-flame ignition will be addressed below by considering the temporal evolution of an unstrained mixing layer formed between two semi-infinite spaces of hydrogen and air. Numerical integrations of the conservation equations of mass, energy, and species, with a detailed scheme employed to evaluate the chemical reactions, will be complemented with analytic studies using the previous two-step reduced chemistry in combination with activation energy asymptotics, the approach taken previously in the analysis of homogeneous ignition [3]. As seen below, one advantage of the analytical approach over direct numerical evaluations is that it readily increases physical understanding by reducing the ignition-time dependence to fewer non-dimensional parameters that define scalings.

As a result of the strong reaction-rate temperature sensitivity, many of the characteristics observed in the classical mixing-layer ignition analysis of Liñán and Crespo [5], which employed a onestep Arrhenius reaction for the chemistry description, emerge also in hydrogen-air mixing-layer ignition below crossover. In particular, as in the previous work [5], when the temperatures of the two sides are very close, the reaction is distributed all across the mixing layer, whereas for hot-air ignition the reaction zone is thin and sits next to the hot boundary. The corresponding analytical treatments, involving either parabolic partial differential equations for describing the evolution with time of the distributed reaction or a twopoint boundary-value problem for hot-side ignition, are also mathematically similar to those encountered previously with one-step Arrhenius chemistry, thereby demonstrating the close applicability of the early general theory to a particular case of technological importance.

\section{Formulation}

Consider the temporal evolution of two stagnant spaces of $\mathrm{H}_{2}$ and air that begin to mix and react at the initial instant of time $t^{\prime}=0$, with the air occupying initially the semispace $x>0$. To describe the ignition process it is convenient to formulate the problem in terms of the dimensionless coordinate $\eta=x /\left(D_{\infty}^{\prime} t^{\prime}\right)^{1 / 2}$, where $D_{\infty}^{\prime}$ represents the value of the $\mathrm{H}_{2}$-air binary diffusion coefficient $D_{\mathrm{H}_{2}-\mathrm{A}}^{\prime}$ evaluated at the air-side temperature. Correspondingly, the transverse velocity $v^{\prime}$ and the diffusion velocity $V_{i}^{\prime}$ of each species $i$ are scaled with $\left(D_{\infty}^{\prime} / t^{\prime}\right)^{1 / 2}$, yielding the dimensionless variables $v=v^{\prime} /\left(D_{\infty}^{\prime} / t^{\prime}\right)^{1 / 2}$ and $V_{i}=V_{i}^{\prime} /\left(D_{\infty}^{\prime} / t^{\prime}\right)^{1 / 2}$. The temperature, density, thermal conductivity, and specific heat at constant pressure are scaled with the properties on the air side to give the dimensionless variables $T=T^{\prime} / T_{\infty}^{\prime}, \quad \rho=\rho^{\prime} / \rho_{\infty}^{\prime}, \quad \lambda=\lambda^{\prime} / \lambda_{\mathrm{A}_{\infty}}^{\prime}$, and $c_{p}=c_{p}^{\prime} / c_{p_{\infty}}^{\prime}$. The problem reduces to that of integrating

$\frac{\partial \rho}{\partial t^{\prime}}+\frac{1}{t^{\prime}}\left[(\rho v)_{\eta}-\frac{1}{2} \eta \rho_{\eta}\right]=0$

$\rho \frac{\partial Y_{i}}{\partial t^{\prime}}+\frac{1}{t^{\prime}}\left[\rho\left(v-\frac{\eta}{2}\right) Y_{i_{\eta}}+\left(\rho Y_{i} V_{i}\right)_{\eta}\right]=\frac{M_{i} \omega_{i}}{\rho_{\infty}^{\prime}}$,

$$
\begin{aligned}
& \rho c_{p} \frac{\partial T}{\partial t^{\prime}}+\frac{1}{t^{\prime}}\left[\rho c_{p}\left(v-\frac{\eta}{2}\right) T_{\eta}-L\left(\lambda T_{\eta}\right)_{\eta}+\rho T_{\eta} \sum_{i} Y_{i} V_{i} c_{p_{i}}\right] \\
& \quad=-\sum_{i} \frac{h_{i} \omega_{i}}{\rho_{\infty}^{\prime} c_{p_{\infty}}^{\prime} T_{\infty}^{\prime}},
\end{aligned}
$$

with initial conditions at $t^{\prime}=0$

$\eta>0: Y_{i}-Y_{i_{\infty}}=T-1=0$,

$\eta<0: Y-Y_{i_{-\infty}}=T-T_{-\infty}=0$,

and boundary conditions for $t^{\prime}>0$ given by

$\eta \rightarrow+\infty: v=Y_{i}-Y_{i_{\infty}}=T-1=0$,

$\eta \rightarrow-\infty: Y-Y_{i_{-\infty}}=T-T_{-\infty}=0$,

with $T_{-\infty}=T_{-\infty}^{\prime} / T_{\infty}^{\prime}$ representing the hydrogen-to-air temperature ratio, which is typically smaller than unity in gas-turbine applications. Here, the subscript $\eta$ is used to denote differentiation with respect to this variable. In the formulation, $Y_{i}, M_{i}, \omega_{i}$, and $h_{i}$, represent, respectively, the mass fraction, molecular mass, chemical production rate (mols per unit volume per unit time), and molar enthalpy of species $i$. The specific heat at constant pressure $c_{p_{i}}^{\prime}$ of species $i$ is scaled with $c_{p_{\infty}}^{\prime}$ to give $c_{p_{i}}=c_{p_{i}}^{\prime} / c_{p_{\infty}}^{\prime}$, a function of the local temperature. The equation of state takes the non-dimensional form

$\rho T \sum_{i} \frac{M_{\mathrm{A}}}{M_{i}} Y_{i}=1$,

where $M_{\mathrm{A}}$ denotes the mean molecular mass of air. With the scaling selected for the transverse coordinate, the Lewis number $L=\lambda_{\mathrm{A}_{\infty}}^{\prime} /\left(\rho_{\infty}^{\prime} c_{p_{\infty}}^{\prime} D_{\infty}^{\prime}\right)$ appears as a factor in (3), which is written with Dufour effects neglected. In the numerical integrations, NASA polynomial fits were used to evaluate specific enthalpies and values of $c_{p_{i}}$, while standard mixture-averaged methods [6] were used to evaluate the multicomponent transport properties, including the thermal conductivity $\lambda$ and the mixture-averaged diffusion coefficients $D_{i}=D_{i}^{\prime} / D_{\infty}^{\prime}$ in the presumed Fickian description of the diffusion velocities $V_{i}=-D_{i}\left(Y_{i}\right)_{\eta} / Y_{i}$, with Soret diffusion included when computing the diffusion velocities of $\mathrm{H}$ and $\mathrm{H}_{2}$. The computations employed the so-called San Diego Mechanism [7], a detailed chemistry description involving 21 reversible elementary reactions and eight reactive species, namely, $\mathrm{O}_{2}, \mathrm{H}_{2}, \mathrm{H}_{2} \mathrm{O}, \mathrm{H}, \mathrm{O}, \mathrm{OH}, \mathrm{HO}_{2}$, and $\mathrm{H}_{2} \mathrm{O}_{2}$.

Note that an arbitrary condition of zero displacement on the air side has been used in writing the boundary condition for $v$. The resulting ignition time is independent of this choice, in that introduction of a different condition, such as zero displacement $v=0$ on the hydrogen side $\eta \rightarrow-\infty$, would cause a shift in the location of the different profiles, but would not modify the resulting ignition time.

\section{Numerical results}

The problem was integrated numerically to determine the transient mixing-layer evolution for different pressures and temperatures below crossover. The use of the stretched variable $\eta$ in place of the original $x$ reduces numerical stiffness for small times, when the physical thickness of the mixing layer is very small. A finite-difference marching scheme was employed to integrate in time, with second-order discretization in $\eta$. A slowly varying non-uniform grid extending for $-10<\eta<10$ was used, with smaller grid spacing in the reactive region, including minimum values of the order of $\delta \eta \lesssim 10^{-2}$, corresponding in physical space to values $\delta x \lesssim 10^{-4} \mathrm{~m}$ for the longest ignition events computed. Smaller marching steps were used as ignition was approached, with time steps reducing to values below $\delta t^{\prime}=10^{-6} \mathrm{~s}$ to capture the localized thermal runaway. Several grid-dependence tests were carried out 


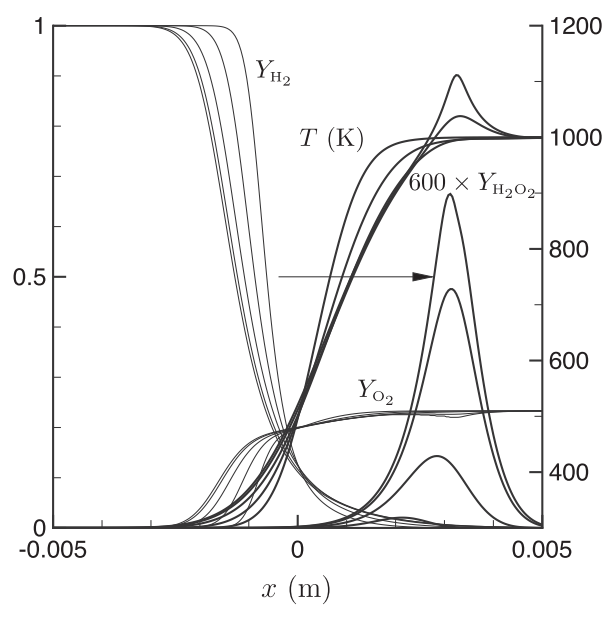

Fig. 1. Profiles of temperature and $\mathrm{H}_{2}, \mathrm{O}_{2}$, and $\mathrm{H}_{2} \mathrm{O}_{2}$ mass fraction obtained at $t^{\prime}=(1.05,2,3,3.5,3.57) \times 10^{-2} \mathrm{~s}$ by integration of $(1)-(8)$ with detailed chemistry for $p=10 \mathrm{~atm}, T_{\infty}^{\prime}=1000 \mathrm{~K}$, and $T_{-\infty}^{\prime}=300 \mathrm{~K}$.

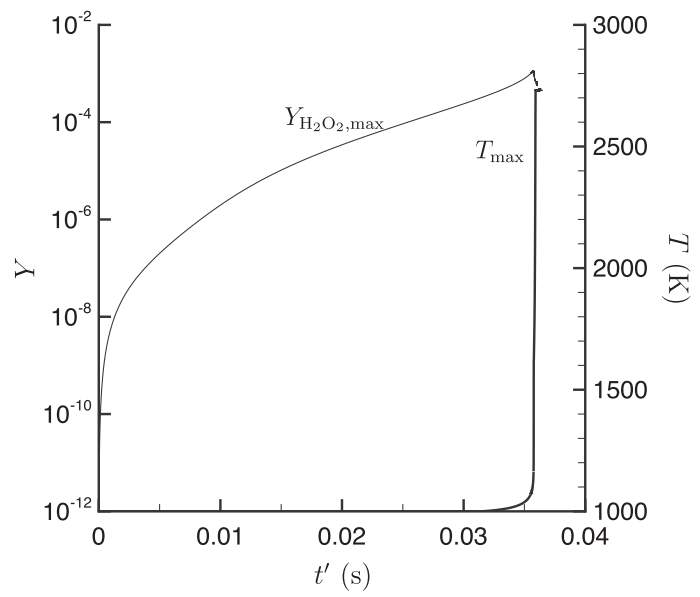

Fig. 2. The evolution with time of of the peak values of $\mathrm{H}_{2} \mathrm{O}_{2}$ mass fraction $\mathrm{Y}_{\mathrm{H}_{2} \mathrm{O}_{2} \text {,max }}$ and temperature $T_{\max }$ obtained by integration of (1)-(8) with detailed chemistry for $T_{\infty}^{\prime}=1000 \mathrm{~K}, T_{\infty}^{\prime}=300 \mathrm{~K}$, and $p=10 \mathrm{~atm}$.

in both space and time to ensure that the results are independent of the selection of scales for the numerical discretization.

The typical transient evolution of the mixing layer found from the numerical integrations is illustrated in Figs. 1 and 2. In particular, Fig. 1 shows profiles of temperature and species across the mixing layer at five different instants of time for $p=10 \mathrm{~atm}$, $T_{\infty}^{\prime}=1000 \mathrm{~K}$, and $T_{-\infty}^{\prime}=300 \mathrm{~K}$. A precipitous increase of temperature and $\mathrm{H}_{2} \mathrm{O}_{2}$ mass fraction is seen to appear after a fairly long period of slow chemical reaction. To further illustrate this rapid increase, the time histories of the corresponding peak values of the $\mathrm{H}_{2} \mathrm{O}_{2}$ mass fraction and of the temperature are shown in Fig. 2. The sudden temperature rise at a well-defined instant of time is indicative of a thermal runaway event, also observed in homogeneous ignition [3].

Several different criteria can be employed to define the ignition time $t_{i}^{\prime}$ precisely. Options include selecting a threshold value for the reactant consumption rate or for the peak value of a given chemical intermediate, with differences in resulting values of $t_{i}^{\prime}$ being typically very small, as is to be expected given the rapid changes observed at ignition. In the present work, the ignition time is defined in connection with the maximum temperature observed across the mixing layer, a function of time that shows an inflection point at a given instant, taken here to be the ignition time. Result-

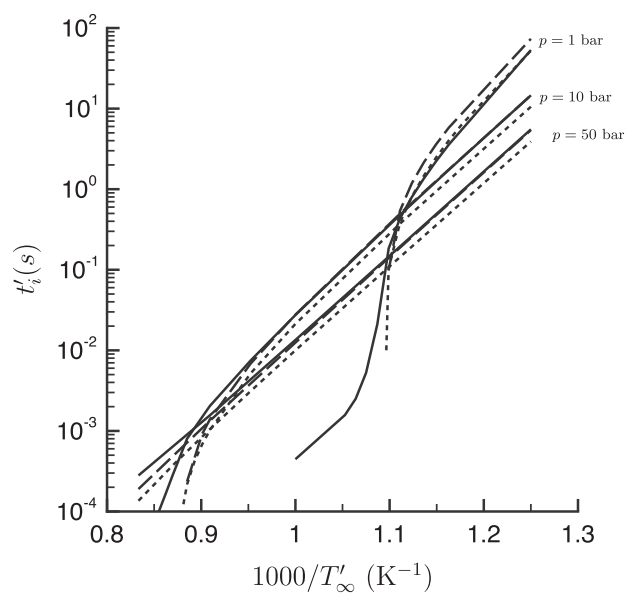

Fig. 3. The variation with air-side temperature of the ignition time in the hydrogenair mixing layer with $T_{\infty}^{\prime}-T_{-\infty}^{\prime}=200 \mathrm{~K}$ as obtained for three different pressures by integration of (1)-(8) with detailed chemistry (solid curves) and 2-step reduced chemistry (long-dashed curves) and by evaluation of the large-activation-energy results (short-dashed curves).

Table 1

Rate coefficients in Arrhenius form $k=A T^{n} \exp \left[-E /\left(R^{o} T^{\prime}\right)\right]$ for the skeletal mechanism.

\begin{tabular}{|c|c|c|c|c|c|}
\hline & Reaction & & $A^{\mathrm{a}}$ & $n$ & $E^{\mathrm{a}}$ \\
\hline 1 & $\mathrm{H}+\mathrm{O}_{2} \rightarrow \mathrm{OH}+\mathrm{O}$ & & $3.52 \times 10^{16}$ & -0.7 & 71.42 \\
\hline \multirow[t]{2}{*}{4} & $\mathrm{H}+\mathrm{O}_{2}+\mathrm{M} \rightarrow \mathrm{HO}_{2}+\mathrm{M}^{\mathrm{b}}$ & $k_{0}$ & $5.75 \times 10^{19}$ & -1.4 & 0.0 \\
\hline & & $k_{\infty}$ & $4.65 \times 10^{12}$ & 0.44 & 0.0 \\
\hline 5 & $\mathrm{H}_{2}+\mathrm{O}_{2} \rightarrow \mathrm{HO}_{2}+\mathrm{H}$ & & $2.69 \times 10^{12}$ & 0.36 & 231.86 \\
\hline 6 & $2 \mathrm{HO}_{2} \rightarrow \mathrm{H}_{2} \mathrm{O}_{2}+\mathrm{O}_{2}$ & & $3.0210^{12}$ & 0.0 & 5.8 \\
\hline 7 & $\mathrm{HO}_{2}+\mathrm{H}_{2} \rightarrow \mathrm{H}_{2} \mathrm{O}_{2}+\mathrm{H}$ & & $1.62 \times 10^{11}$ & 0.61 & 100.14 \\
\hline \multirow[t]{2}{*}{8} & $\mathrm{H}_{2} \mathrm{O}_{2}+\mathrm{M} \rightarrow 2 \mathrm{OH}+\mathrm{M}^{\mathrm{c}}$ & $k_{0}$ & $8.15 \times 10^{23}$ & -1.9 & 207.62 \\
\hline & & $k_{\infty}$ & $2.62 \times 10^{19}$ & -1.39 & 214.74 \\
\hline
\end{tabular}

a Units are mol, $\mathrm{s}, \mathrm{cm}^{3}, \mathrm{~kJ}$, and $\mathrm{K}$.

b Chaperon efficiencies are 2.5 for $\mathrm{H}_{2}, 16.0$ for $\mathrm{H}_{2} \mathrm{O}, 0.7$ for $\mathrm{Ar}$ and $\mathrm{He}$ and 1.0 for all other species; Troe falloff with $F_{c}=0.5$.

c Chaperon efficiencies are 2.0 for $\mathrm{H}_{2}, 6.0$ for $\mathrm{H}_{2} \mathrm{O}, 0.4$ for $\mathrm{Ar}$ and $\mathrm{He}$ and 1.0 for all other species; $F_{c}=0.265 \exp (-T / 94 \mathrm{~K})+0.735 \exp (-T / 1756 \mathrm{~K})+\exp (-5182 \mathrm{~K} / T)$.

ing ignition times obtained for different pressures and different boundary temperatures are shown in Fig. 3, where the solid curves represent the numerical results for the variation of $t_{i}^{\prime}$ with the airside temperature $T_{\infty}^{\prime}$ for three different pressures, with the air-tohydrogen temperature difference being kept at a constant value $T_{\infty}^{\prime}-T_{-\infty}^{\prime}=200 \mathrm{~K}$.

Besides results of detailed-chemistry computations, Fig. 3 also shows as long-dashed curves predictions of ignition times obtained numerically with the two-step reduced-chemistry description previously developed for homogeneous ignition [3]. The previous mechanism can be more conveniently expressed by writing separate steps for water vapor and hydrogen peroxide production, according to

$2 \mathrm{H}_{2}+\mathrm{O}_{2} \stackrel{\mathrm{I}}{\rightarrow} 2 \mathrm{H}_{2} \mathrm{O}$

$\mathrm{H}_{2}+\mathrm{O}_{2} \stackrel{\text { II }}{\rightarrow} \mathrm{H}_{2} \mathrm{O}_{2}$,

with rates given by

$\omega_{\mathrm{I}}=\frac{1+\alpha}{1-\alpha} k_{8} C_{\mathrm{M}_{8}} C_{\mathrm{H}_{2} \mathrm{O}_{2}}$,
$\omega_{\mathrm{II}}=\frac{k_{7} k_{8}^{1 / 2}}{k_{6}^{1 / 2}} \frac{C_{\mathrm{H}_{2}} C_{\mathrm{M}_{8}}}{(1-\alpha)^{3 / 2}}\left[\left(1-\frac{\alpha}{2}\right) \frac{k_{5} C_{\mathrm{H}_{2}} \mathrm{C}_{\mathrm{O}_{2}}}{k_{8} C_{\mathrm{M}_{8}}^{2}}+\frac{C_{\mathrm{H}_{2} \mathrm{O}_{2}}}{C_{\mathrm{M}_{8}}}\right]^{1 / 2}$,

in terms of the reaction-rate constants of the elementary reactions shown in Table 1 . The reaction-rate ratio $\alpha=2 k_{1} /\left(k_{4} C_{\mathrm{M}_{4}}\right)$ appearing here, which equals unity at crossover, decreases rapidly as the 
temperature decreases, reaching values on the order of $\alpha \sim 0.05$ as the temperature falls $200 \mathrm{~K}$ below crossover. Here, $C_{i}=\rho^{\prime} Y_{i} / M_{i}$ is the concentration of species $i$, with $C_{\mathrm{M}_{4}}$ and $C_{\mathrm{M}_{8}}$ representing the effective third-body concentrations for reactions 4 and 8, which, for the ignition problem considered here, can be evaluated according to $C_{\mathrm{M}_{4}}=C_{\mathrm{M}}(1+1.5 X)$ and $C_{\mathrm{M}_{8}}=C_{\mathrm{M}}(1+X)$ in terms of the $\mathrm{H}_{2}$ mole fraction $X$ and the third-body concentration $C_{M}=\rho^{\prime} \sum_{i} Y_{i} / M_{i}$. It can be seen in Fig. 3 that the two-step reduced chemistry gives satisfactory predictions for the mixing-layer ignition time for all conditions of pressure and temperature below crossover, with levels of accuracy comparable to those previously obtained in homogeneous ignition computations [3].

Because of the relative simplicity of the laminar problem considered here, the computational time was not a limiting factor in the numerical integrations. Nevertheless, it is worth mentioning that use of the two-step kinetics reduces the required computational time by a factor of about two without affecting significantly computational stiffness, the reduction being a direct consequence of the smaller number of conservation equations for species involved in the simplified-chemistry description. This significant decrease in computational time would be of interest in direct numerical simulations of ignition in turbulent environments, for which use of reduced mechanisms has proved to be successful for temperatures above crossover [8]. Note that the saving in computational time associated with the use of reduced chemistry is significantly larger in computations employing multi-component diffusion models instead of standard mixture-averaged diffusivities, because of the resulting decrease in size of the corresponding diffusion matrix [9].

\section{General character of the theoretical analysis of ignition}

As seen in the numerical integrations, ignition in hydrogen-air mixing layers at temperatures below crossover takes place as a thermal explosion, thereby reproducing the behavior previously observed in homogeneous ignition [3]. Many of the results of the seminal activation-energy analysis of Liñán and Crespo [5], developed with a generic one-step irreversible reaction with Arrhenius rate for the chemistry description, can be used in the present context, the main differences with hydrogen stemming from the underlying chemistry description and the fuel diffusivity. In particular, as in [5] the chemical reaction in hydrogen-air mixing layers is very weak prior to ignition, so that in the first approximation the reactants and temperature are given by the self-similar solutions corresponding to chemically frozen mixing, to be described next in Section 5.

It is shown in Section 6 that the thermal runaway can be analyzed on the basis of the two-step reduced-chemistry description [3] by considering the small temperature perturbations associated with chemical reaction, whose evolution is coupled to that of the $\mathrm{H}_{2} \mathrm{O}_{2}$ concentration. While the analysis of that section takes advantage of simplifications that arise when the initial fuel and oxidizer temperatures are nearly equal, numerical integration of two parabolic partial differential equations is still needed, which is more complicated but mathematically similar to that encountered by Liñán and Crespo [5]. A simpler solution appears when, as typically occurs in gas-turbine applications, the air-to-hydrogen temperature difference is larger than the air-side temperature by a factor exceeding the reciprocal of the controlling non-dimensional activation energy, as is shown in Section 7. That analysis leads to a diffusion-reaction problem in which the non-dimensional time enters as an effective Damköhler number, with the ignition time determined as the critical value above which a solution cannot exist.

\section{The chemically frozen mixing layer}

In the absence of chemical reaction, the solution is self-similar when expressed in terms of the variable $\eta$. The only chemical species present in the mixture are $\mathrm{H}_{2}, \mathrm{O}_{2}$ and $\mathrm{N}_{2}$. The solution can be simplified by neglecting differences between $\mathrm{O}_{2}$ and $\mathrm{N}_{2}$, that is, treating air as a single species. This approximation causes the mixture to be effectively binary, with the result that all local properties can be characterized in terms of the chemically frozen values of the $\mathrm{H}_{2}$ mass fraction and temperature, which will be denoted by $Y$ and $T_{f}$ in the following. For instance, the equation of state (8) reduces to

$\rho T_{f}=\frac{1}{\mathcal{M} Y+1-Y}$,

with $\mathcal{M}=M_{\mathrm{A}} / M_{\mathrm{H}_{2}}$ representing the air-to-hydrogen molecularmass ratio, while the thermal conductivity can be accurately approximated by

$\lambda=\frac{1+\left(\Lambda \mathcal{M}^{2 / 3}-1\right) Y}{1+\left(\mathcal{M}^{2 / 3}-1\right) Y} T_{f}^{\sigma}$,

an expression suggested by Rosner for gaseous binary mixtures [10], with $\Lambda=\lambda_{\mathrm{H}_{2 \infty}}^{\prime} / \lambda_{\mathrm{A}_{\infty}}^{\prime}$ denoting here the hydrogen-to-air thermal conductivity ratio evaluated at the air-side temperature. On the other hand, it is known that for a binary mixture the multicomponent transport equation derived from the kinetic theory can be solved explicitly [11]. Neglecting barodiffusion and using -0.29 for the Soret factor [12] yields

$Y V_{\mathrm{H}_{2}}=-(1-Y) V_{\mathrm{A}}=-T_{f}^{1+\sigma}\left(Y_{\eta}-0.29 Y T_{f_{\eta}} / T_{f}\right)$

for the dimensionless diffusion velocities of hydrogen and air. In (12) and (13) the same exponent $\sigma$ is employed for the presumed power-law temperature dependence of the transport coefficients. An additional simplification emerges by noting that the specific heats per mol of the different species $\mathrm{H}_{2}, \mathrm{O}_{2}$ and $\mathrm{N}_{2}$ differ only by a small amount in the temperature range considered ( $300 \mathrm{~K}<T^{\prime}<1300 \mathrm{~K}$ ), so that to write the energy Eq. (3) for the binary mixture one may use the approximations $\rho c_{p}=1 / T_{f}$ and $\sum_{i} Y_{i} V_{i} c_{p_{i}}=(\mathcal{M}-1) Y V_{\mathrm{H}_{2}}$, finally reducing the problem to that of integrating

$(\rho v)_{\eta}-\frac{1}{2} \eta \rho_{\eta}=0$
$\left(\rho Y V_{\mathrm{H}_{2}}\right)_{\eta}+\rho\left(v-\frac{1}{2} \eta\right) Y_{\eta}=0$
$L\left(\lambda T_{f_{\eta}}\right)_{\eta}-(\mathcal{M}-1) \rho Y V_{\mathrm{H}_{2}} T_{f_{\eta}}-\frac{1}{T_{f}}\left(v-\frac{1}{2} \eta\right) T_{f_{\eta}}=0$

supplemented with (11)-(13) and with boundary conditions

$\eta \rightarrow+\infty: v=Y=T_{f}-1=0$,

$\eta \rightarrow-\infty: Y-1=T_{f}-T_{-\infty}=0$.

Using the properties of hydrogen and air provides the numerical values $\mathcal{M}=14.5, \Lambda=7, \sigma=0.7$ and $L=0.3$ for the different constants appearing in (11), (12), and (16), thereby leaving the hydrogen-to-air temperature ratio $T_{-\infty}$ emerging in (18) as the only parameter to be varied in the computations.

In general, numerical integration is needed for the solution, with sample profiles of $T_{f}$ and $Y$ shown in Fig. 4 for $T_{-\infty}=0.9$. As shown in $[13,14]$, an analytic solution arises for the quasi-isothermal case $\left|T_{-\infty}-1\right| \ll 1$, when $\left|T_{f}-1\right| \ll 1$ everywhere across the mixing layer. In that case, the diffusion velocity given in (13) reduces to $Y V_{\mathrm{H}_{2}}=-Y_{\eta}$ whereas differentiation of (11) yields $\rho Y_{\eta}=-\rho_{\eta} /[(\mathcal{M}-1) \rho]$. Substituting these last two expressions into (15) gives 


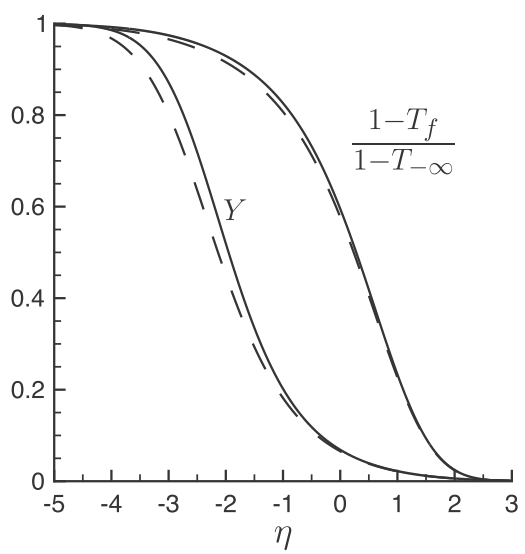

Fig. 4. The frozen profiles of temperature and hydrogen mass fraction determined from (14)-(18) for $T_{-\infty}=0.9$ (solid curves) and evaluated from the quasi-isothermal solution given in (21) and (25) (dashed curves).

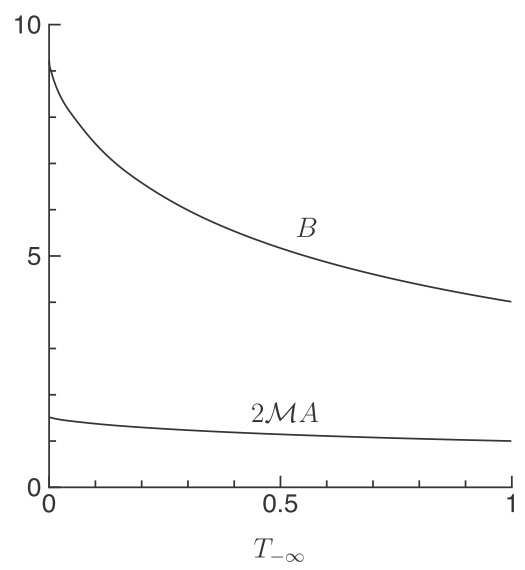

Fig. 5. The variation with $T_{-\infty}$ of the factors $A$ and $B$ corresponding to the asymptotic behaviors given in (26).

$\rho\left(\rho_{\eta} / \rho\right)_{\eta}-\left(v-\frac{1}{2} \eta\right) \rho_{\eta}=0$

thereby providing

$\left(\rho_{\eta} / \rho+v\right)_{\eta}=0$,

after addition of (14). Using the boundary conditions on the air side in integrating once (20) yields $\rho v=-\rho_{\eta}$, which can be employed to rewrite (14) as $\rho_{\eta \eta}+\frac{1}{2} \eta \rho_{\eta}=0$, a linear equation that can be finally integrated with the conditions $\rho(\infty)-1=\rho(-\infty)-1 / \mathcal{M}=0$ to give

$\rho=\frac{1}{(\mathcal{M}-1) Y+1}=1-\frac{\mathcal{M}-1}{2 \mathcal{M}} \operatorname{erfc}(\eta / 2)$.

In this description, the resulting gas velocity and hydrogen diffusion velocity can be computed with use made of the accompanying equations

$v=-(\mathcal{M}-1) \rho Y V_{\mathrm{H}_{2}}=-\rho_{\eta} / \rho$,

that follow from the above derivation. It is of interest that in the quasi-isothermal solution the hydrogen mole fraction

$X=\frac{\mathcal{M} Y}{\mathcal{M} Y+1-Y}$

is simply given by
$X=\frac{1}{2} \operatorname{erfc}(\eta / 2)$

as implied by the second equation in (21). These results can be used to compute the small value of $1-T_{f}$ by integrating $L\left(\lambda T_{f_{\eta}}\right)_{\eta}+(\eta / 2) T_{f_{\eta}}=0$, obtained from (16) with use made of (22). The resulting temperature distribution can be written in the form

$\frac{1-T_{f}}{1-T_{-\infty}}=\frac{\int_{\eta}^{+\infty} \lambda^{-1} \exp \left[-\int_{0}^{\tilde{\eta}} \bar{\eta} /(2 L \lambda) \mathrm{d} \bar{\eta}\right] \mathrm{d} \tilde{\eta}}{\int_{-\infty}^{+\infty} \lambda^{-1} \exp \left[-\int_{0}^{\tilde{\eta}} \bar{\eta} /(2 L \lambda) \mathrm{d} \bar{\eta}\right] \mathrm{d} \tilde{\eta}}$,

where the function $\lambda$ is to be evaluated from (12) with $T_{f}=1$. The accuracy of the approximate expressions (21) and (25) is tested in Fig. 4 for $1-T_{-\infty}=0.1$. As can be seen, reasonably good agreement is obtained, with the analytic expressions being increasingly accurate as the air side is approached.

In gas-turbine applications the air side is hotter (i.e., $T_{-\infty}<1$ ) and ignition tends to occur for $\eta \gg 1$, where the distribution of the chemically frozen profiles of hydrogen and temperature are given in the first approximation by

$Y=A \operatorname{erfc}\left(\frac{\eta}{2}\right)$ and $\frac{1-T_{f}}{1-T_{-\infty}}=B \operatorname{erfc}\left(\frac{\eta}{2 \sqrt{L}}\right)$

as can be obtained by integration of $Y_{\eta \eta}+\frac{1}{2} \eta Y_{\eta}=0$ and $L\left(T_{f}\right)_{\eta \eta}+\frac{1}{2} \eta\left(T_{f}\right)_{\eta}=0$, the asymptotic air-side form of (15) and (16), respectively. Values of $A$ and $B$, to be used later when analyzing ignition, are shown below in Fig. 5 as a function of $T_{-\infty}$. For $1-T_{-\infty} \ll 1$, these constants take the values $A=1 /(2 \mathcal{M})$ and $B=4.01$, as can be computed from (21) and (25).

\section{The reduced ignition problem for $1-T_{-\infty} \lesssim \beta^{-1}$}

Ignition can be described by studying the evolution of the small values of the temperature increment from the chemically frozen state $T-T_{f}$ and the concentration of $\mathrm{H}_{2} \mathrm{O}_{2}$, needed to evaluate the reaction rates given in (10). When the hydrogen-side temperature differs from the air-side temperature by a small amount $1-T_{-\infty} \lesssim \beta^{-1} \ll 1$ the chemical reaction proceeds at a comparable rate all across the mixing layer [5]. For the analysis of this case, we begin by writing the conservation equations (2) and (3) for the two-step reduced mechanism, yielding

$$
\begin{aligned}
& \rho \frac{\partial Y_{\mathrm{H}_{2} \mathrm{O}_{2}}}{\partial t^{\prime}}+\frac{1}{t^{\prime}}\left[\rho\left(v-\frac{\eta}{2}\right)\left(Y_{\mathrm{H}_{2} \mathrm{O}_{2}}\right)_{\eta}+\left(\rho Y_{\mathrm{H}_{2} \mathrm{O}_{2}} V_{\mathrm{H}_{2} \mathrm{O}_{2}}\right)_{\eta}\right] \\
& =\frac{M_{\mathrm{H}_{2} \mathrm{O}_{2}}}{\rho_{\infty}^{\prime}} \omega_{\mathrm{II}}
\end{aligned}
$$

and

$\frac{\partial T}{\partial t^{\prime}}-\frac{1}{t^{\prime}}\left[\frac{\eta}{2} T_{\eta}+L\left(\lambda T_{\eta}\right)_{\eta}\right]=-\frac{2 h_{\mathrm{H}_{2} \mathrm{O}}}{\rho_{\infty}^{\prime} c_{p_{\infty}}^{\prime} T_{\infty}^{\prime}} \omega_{\mathrm{I}}$,

with the heat release associated with $\mathrm{H}_{2} \mathrm{O}_{2}$ production being neglected in the energy balance, an excellent approximation as previously noted [3]. Because of the condition (22) that applies for $1-T_{\infty} \ll 1$, the convective and enthalpy flux terms in (3) cancel and do not appear in (28).

In writing the diffusive flux of $\mathrm{H}_{2} \mathrm{O}_{2}$ appearing in (27) one may employ the Fickian description derived in the appendix for diffusion of a minor species into a binary mixture of $\mathrm{H}_{2}$ and air. The corresponding Eqs. (A-5) and (A-6) can be written for $\mathrm{H}_{2} \mathrm{O}_{2}$ in the dimensionless form

$Y_{\mathrm{H}_{2} \mathrm{O}_{2}} V_{\mathrm{H}_{2} \mathrm{O}_{2}}=-\frac{L}{L_{\mathrm{H}_{2} \mathrm{O}_{2}}} \mathcal{D}\left(Y_{\mathrm{H}_{2} \mathrm{O}_{2}}\right)_{\eta}$,

in terms of the air-side value of the $\mathrm{H}_{2} \mathrm{O}_{2}$ Lewis number 
$L_{\mathrm{H}_{2} \mathrm{O}_{2}}=\left(\frac{\lambda_{\mathrm{A}}^{\prime}}{\rho^{\prime} C_{p}^{\prime} D_{\mathrm{H}_{2} \mathrm{O}_{2}-\mathrm{A}}^{\prime}}\right)_{\infty}$

whose value is found to be $L_{\mathrm{H}_{2} \mathrm{O}_{2}}=1.1$, and the dimensionless diffusivity

$\mathcal{D}=\frac{1+(\mathcal{M}-1) Y}{1+(\mathcal{M} \Delta-1) Y}$,

a function of the composition to be evaluated with $\Delta=D_{\mathrm{H}_{2} \mathrm{O}_{2}-\mathrm{A}}^{\prime} / D_{\mathrm{H}_{2} \mathrm{O}_{2}-\mathrm{H}_{2}}^{\prime}=0.261$ used for the ratio of binary diffusion coefficients.

As shown in [3], the activation energies of the effective reaction-rate constants $k_{8}$ and $k_{7} k_{8}^{1 / 2} / k_{6}^{1 / 2}$ appearing in (10) differ only by a small relative amount, so that in writing the Frank-Kamenetskii linearizations about the air-side temperature

$k_{8}=\left(k_{8}\right)_{\infty} \exp [\beta(T-1)]$ and

$$
\frac{k_{7} k_{8}^{1 / 2}}{k_{6}^{1 / 2}}=\left(\frac{k_{7} k_{8}^{1 / 2}}{k_{6}^{1 / 2}}\right)_{\infty} \exp [\beta(T-1)],
$$

one may employ a single value $\beta=E_{8} /\left(R^{0} T_{\infty}^{\prime}\right)+n_{8}$ for the nondimensional activation energy, defined for definiteness with the reaction-rate parameters of the elementary reaction 8 , to give for instance $\beta=29.31$ at $T_{\infty}^{\prime}=800 \mathrm{~K}$. The product $\beta(T-1)$ in the above exponential factors can be expressed in terms of the rescaled temperature increment from the chemically frozen state $\theta=\beta\left(T-T_{f}\right)$ according to

$\beta(T-1)=\beta\left(T-T_{f}\right)-\beta\left(1-T_{f}\right)=\theta-\theta_{-\infty} \Theta_{f}$,

with

$\theta_{-\infty}=\beta\left(1-T_{-\infty}\right) \quad$ and $\quad \Theta_{f}=\frac{1-T_{f}}{1-T_{-\infty}}$.

To write (27) and (28) in dimensionless form we further introduce a normalized hydrogen-peroxide mass fraction

$$
\begin{aligned}
\varphi= & \left(1-\alpha_{\infty}\right)^{1 / 3}\left(1+\alpha_{\infty}\right)^{2 / 3}(q \beta)^{2 / 3} \mathcal{M}^{1 / 3} \\
& \times \frac{M_{\mathrm{H}_{2}}}{M_{\mathrm{H}_{2} \mathrm{O}_{2}}}\left(\frac{\left(k_{6} k_{8}\right)^{1 / 3}}{k_{7}^{2 / 3}}\right)_{\infty} Y_{\mathrm{H}_{2} \mathrm{O}_{2}}
\end{aligned}
$$

along with the rescaled time

$t=\frac{\left(1+\alpha_{\infty}\right)^{1 / 3}}{\left(1-\alpha_{\infty}\right)^{4 / 3}}(q \beta)^{1 / 3} \mathcal{M}^{2 / 3}\left(\frac{\left(k_{7} k_{8}\right)^{2 / 3} C_{\mathrm{M}_{\infty}}}{k_{6}^{1 / 3}}\right)_{\infty} t^{\prime}$

where the non-dimensional heat of reaction

$q=\left(\frac{-2 h_{\mathrm{H}_{2} \mathrm{O}} C_{\mathrm{M}}}{\rho^{\prime} C_{p}^{\prime} T^{\prime}}\right)_{\infty}$

and the crossover parameter

$\alpha_{\infty}=\left(\frac{2 k_{1}}{k_{4} C_{\mathrm{M}}}\right)_{\infty}$

are also evaluated at the air-side temperature.

The problem reduces to that of integrating the two equations

$$
\begin{gathered}
\rho \frac{\partial \varphi}{\partial t}+\frac{1}{t}\left[\rho\left(v-\frac{\eta}{2}\right) \varphi_{\eta}-\frac{L}{L_{\mathrm{H}_{2} \mathrm{O}_{2}}}\left(\rho \mathcal{D} \varphi_{\eta}\right)_{\eta}\right] \\
=\Omega_{\varphi} Y e^{-\theta_{-\infty} \Theta_{f}}\left[a \Omega_{I} Y(1-Y)+\varphi\right]^{1 / 2} e^{\theta}
\end{gathered}
$$

and

$\frac{\partial \theta}{\partial t}-\frac{1}{t}\left[\frac{\eta}{2} \theta_{\eta}+L\left(\lambda \theta_{\eta}\right)_{\eta}\right]=\Omega_{\theta} e^{-\theta_{-\infty} \theta_{f}} \varphi e^{\theta}$,

with inital conditions $\varphi=\theta=0$ at $t=0$ and boundary conditions $\varphi=\theta=0$ as $\eta \rightarrow \pm \infty$. The functions of the composition
$\Omega_{\varphi}=\frac{(1+X)^{1 / 2}\left[1-\left(1-\mathcal{M}^{-1}\right) X\right]^{3 / 2}}{\left[1-\alpha_{\infty} /(1+1.5 X)\right]^{3 / 2} /\left[1-\alpha_{\infty}\right]^{3 / 2}}$,

$\Omega_{\theta}=\frac{(1+X)\left[1-\left(1-\mathcal{M}^{-1}\right) X\right]\left[1+\alpha_{\infty} /(1+1.5 X)\right] /\left[1+\alpha_{\infty}\right]}{\left[1-\alpha_{\infty} /(1+1.5 X)\right] /\left[1-\alpha_{\infty}\right]}$,

$\Omega_{I}=\frac{\left[1-\left(1-\mathcal{M}^{-1}\right) X\right]\left[1-\frac{1}{2} \alpha_{\infty} /(1+1.5 X)\right]}{(1+X)\left(1-\frac{1}{2} \alpha_{\infty}\right)}$,

can be computed at each $\eta$ with use made of the expression $X=\operatorname{erfc}$ $(\eta / 2) / 2$ for the hydrogen mole fraction. The initiation rate is measured by the parameter

$a=\left(1-\frac{\alpha_{\infty}}{2}\right)\left(1-\alpha_{\infty}\right)^{1 / 3}\left(1+\alpha_{\infty}\right)^{2 / 3}(q \beta)^{2 / 3} \mathcal{M}^{1 / 3} \frac{k_{5} k_{6}^{1 / 3}}{\left(k_{7} k_{8}\right)^{2 / 3}} Y_{\mathrm{O}_{2 \infty}}$,

which is evaluated with the air-side temperature. As corresponds to $1-T_{-\infty} \ll 1$, the density $\rho$ and hydrogen mass fraction $Y$ are to be determined from (21), while the frozen temperature distribution $\Theta_{f}=\left(1-T_{f}\right) /\left(1-T_{-\infty}\right)$ is to be computed from (25) and the transport coefficients $\lambda$ and $\mathcal{D}$ are given in (12) and (31), respectively, with $T_{f}=1$ in (12).

The numerical integration of (39) and (40) describes the thermal explosion, including a thermal runaway at a finite ignition time $t_{i}$. Characteristic profiles of $\theta$ obtained for two different values of $\theta_{-\infty}$ are shown in Fig. 6 . The plots are illustrative of the selfaccelerating nature of the temperature growth. It can be seen in Fig. 6 that as the air-to-hydrogen temperature difference $\theta_{-\infty}$ increases, the reaction zone moves towards the air side and becomes thinner, ushering in a regime of hot-side ignition to be analyzed separately in the next section.

Since the value of $a$ is always very small, i.e., $a \sim 10^{-4}$ at $T_{\infty}^{\prime}=800 \mathrm{~K}$, the associated initiation term is only important for
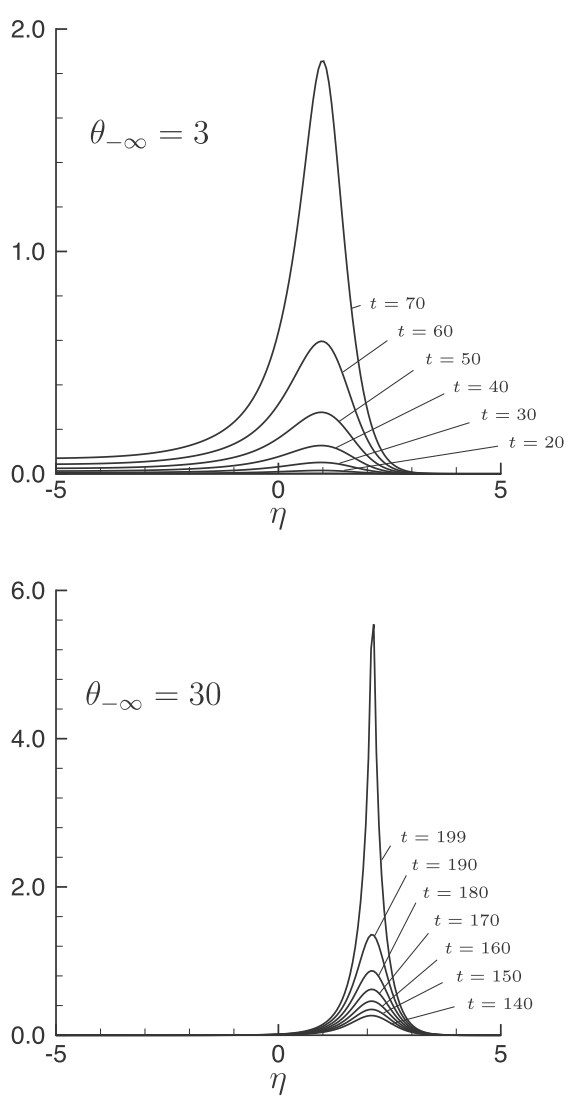

Fig. 6. Profiles of $\theta$ determined from integrations of (39) and (40) for $\theta_{-\infty}=3$ (upper plot) and for $\theta_{-\infty}=30$ (lower plot) with $\alpha_{\infty}=0$ and $a=10^{-4}$. 
$t \sim a^{1 / 2}$ when $\varphi \sim \theta \sim a$ but becomes negligible for $t \gg a^{1 / 2}$. As a result, the ignition time obtained by integrating (39) and (40) is found to be independent of the precise value of $a$ employed in the integration, provided $a \ll 1$. The constant value $a=10^{-4}$ is used in the computations reported here. In view of this simplification, the resulting non-dimensional ignition time $t_{i}$ turns out to be a function of just two parameters, namely, the air-to-hydrogen temperature difference $\theta_{-\infty}$ defined in (34) and the crossover parameter $\alpha_{\infty}$ defined in (38). The associated parametric dependences are summarized in Fig. 7. As expected, the ignition time increases at a fixed air temperature as the crossover temperature is approached (i.e., increasing values of $\alpha_{\infty}$ by decreasing pressure) and also as the hydrogen temperature is reduced (i.e., increasing values of $\theta_{-\infty}$ ). With the scales employed, the functions $\Omega_{\varphi}, \Omega_{\theta}$, and $\Omega_{I}$ defined in (41) approach unity as $X \ll 1$ on the air side, regardless of the value of $\alpha_{\infty}$. Consequently, in the limit of large temperature differences $\theta_{-\infty} \gg 1$, when the reaction migrates towards the air side, the ignition time $t_{i}$ becomes weakly dependent on $\alpha_{\infty}$.

The definition given in (36) can be used to evaluate the dimensional ignition time $t_{i}^{\prime}$ from the results of the activation-energy analysis given in Fig. 7. Sample evaluations are compared in Fig. 3 with the results of the detailed and reduced chemistry computations. It can be seen in Fig. 3 that the agreement is reasonably good, with errors on the order of $20 \%$ that become somewhat larger as crossover is approached, as expected.

\section{The ignition near the air boundary for $1-T_{-\infty} \gg \beta^{-1}$}

When the hydrogen temperature is smaller than the air temperature by an amount $1-T_{-\infty} \gg \beta^{-1}$, corresponding to values of

$\theta_{-\infty}=\beta\left(1-T_{-\infty}\right) \gg 1$,

the analysis given above needs to be modified. As indicated by the results in the lower plot of Fig. 6, in this case of hot-boundary ignition the chemical reaction is no longer distributed across the mixing layer, but it is instead confined within a thin layer located near the air-side boundary, where the frozen temperature differs from the boundary value by a small relative amount of order $1-T_{f} \sim \beta^{-1}$, with the chemical reaction proceeding at an exponentially small rate outside. This limiting case corresponds to the conditions of Fig. 1, for example. The solution simplifies because in this far-field reaction region $v \ll 1$ and $\rho \simeq \lambda \simeq \mathcal{D} \simeq \Omega_{\varphi} \simeq \Omega_{\theta} \simeq 1$, while the

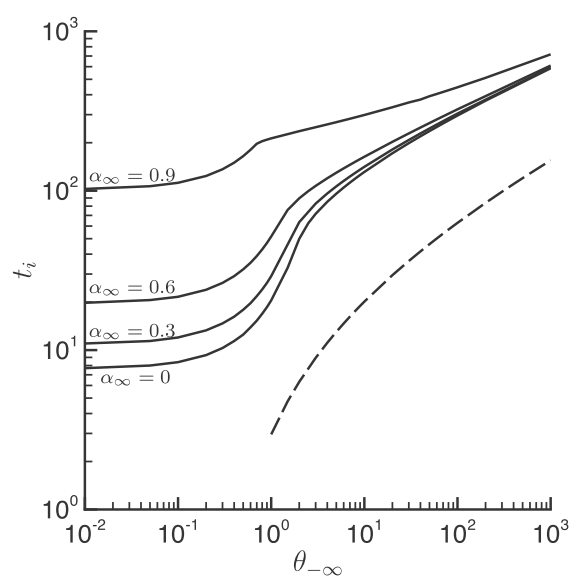

Fig. 7. The variation with $\theta_{-\infty}$ of $t_{i}$ for different values of $\alpha_{\infty}$ as obtained by integrating (39) and (40); the dashed curve represents the prediction of the hot-air asymptotic analysis obtained from evaluating (44), (50), and (52) with $\tau_{i}=0.329$. chemically-frozen profiles of hydrogen mass fraction and temperature are given by (26).

Following the analysis of Liñán and Crespo [5], the condition $1-T_{f}=\beta^{-1}$ (i.e., $\theta_{-\infty} \Theta_{f}=1$ ) is used to determine the location of the reaction layer $\eta_{R}$ according to

$B \operatorname{erfc}\left(\frac{\eta_{R}}{2 \sqrt{L}}\right)=\frac{1}{\theta_{-\infty}}$,

giving a relatively large value

$\eta_{R} \sim\left(4 L \ln \theta_{-\infty}\right)^{1 / 2} \gg 1$,

as can be obtained by using in (44) the asymptotic expansion for large values of the argument of the complementary error function

$\operatorname{erfc}\left(\frac{\eta_{R}}{2 \sqrt{L}}\right) \simeq \frac{2 \sqrt{L}}{\sqrt{\pi} \eta_{R}} \exp \left(-\frac{\eta_{R}^{2}}{4 L}\right)$

and solving for $\eta_{R}$ with $\theta_{-\infty} \gg 1$. Consideration of the limit $\eta_{R} \gg 1$ enables a simplified solution to be derived. In particular, the expansion

$\theta_{-\infty} \Theta_{f} \simeq \exp \left[-\eta_{R}\left(\eta-\eta_{R}\right) /(2 L)\right]$

for the temperature profile (26) about $\eta=\eta_{R}$ indicates that the characteristic thickness of the reaction layer is $\left(\eta-\eta_{R}\right) \sim 1 / \eta_{R} \ll 1$. Also, the expansion suggests the coordinate

$\xi=\exp \left[-\eta_{R}\left(\eta-\eta_{R}\right) /(2 L)\right]$

for describing the reaction layer, which in the first approximation reduces $(26)$ to

$Y=Y_{R} \xi^{L}$ and $\theta_{-\infty} \Theta_{f}=\xi$,

where

$Y_{R}=A \operatorname{erfc}\left(\frac{\eta_{R}}{2}\right)$

is the characteristic value of the $\mathrm{H}_{2}$ mass fraction at $\eta=\eta_{R}$.

Before proceeding with the analysis, it is of interest to analyze the question of whether fuel consumption may exert a significant influence on the ignition process in this hot-boundary case, when ignition occurs far on the oxidizer side, where the amount of fuel available is limited by its diffusion rate across the mixing layer. To assess this effect, one may use (45) and (50) to show that the characteristic value of the hydrogen mass fraction in the reaction layer is of order $Y_{R} \sim \beta^{-L}$ with $L=0.3$. On the other hand, we have seen that, because of the large value of the activation energy, ignition is associated with small temperature increments by the chemical reaction $T-T_{f}$ that are of order $\beta^{-1}$, consuming in the process a small amount of fuel, which is found to be of order $\beta^{-1}$ for $q \sim O(1)$. A straightforward comparison between both estimates indicates that, since $L<1$, in the limit of large activation energies the amount of fuel available in the reaction layer is much larger than the amount needed for ignition to occur, according to $Y_{R} \sim \beta^{-L} \gg \beta^{-1}$. Consequently, in computing ignition one may neglect fuel consumption in the first approximation and use the chemically frozen profile given in the first equation of (49) to evaluate the chemical reaction rate. Note that, in that respect, the ignition process for hydrogen mixing with hot air is simpler than that of fuels with Lewis numbers equal to or larger than unity, which require consideration of fuel consumption in the reaction region, as shown in the equidiffusional case by Liñán and Crespo [5].

The solution further simplifies because the accumulation terms in (27) and (28) are found to be a factor $\eta_{R}^{-2}$ smaller than the diffusion terms. If these unsteady terms are neglected, then the description of the reaction layer reduces to a quasisteady diffusionreaction balance with reactant consumption neglected. The 
problem is conveniently formulated in terms of a modified hydrogen-peroxide concentration

$\Phi=\frac{\varphi}{\left(L_{\mathrm{H}_{2} \mathrm{O}_{2}} Y_{R}\right)^{2 / 3}}$,

and a rescaled time

$\tau=\frac{4 L}{\eta_{R}^{2}}\left(L_{\mathrm{H}_{2} \mathrm{O}_{2}} Y_{R}\right)^{2 / 3} t$

producing the boundary-value problem

$\xi^{2} \frac{\mathrm{d}^{2} \theta}{\mathrm{d} \xi^{2}}+\tau \Phi e^{\theta-\xi}=0$

$\xi^{2} \frac{\mathrm{d}^{2} \Phi}{\mathrm{d} \xi^{2}}-\left(L_{\mathrm{H}_{2} \mathrm{O}_{2}}-1\right) \xi \frac{\mathrm{d} \Phi}{\mathrm{d} \xi}+\tau \xi^{L} \Phi^{1 / 2} e^{\theta-\xi}=0$

to be integrated with boundary conditions

$\theta=\Phi=0$ at $\xi=0$,

as corresponds to matching with the chemical equilibrium solution existing on the air side of the reaction layer, and

$\frac{\mathrm{d} \theta}{\mathrm{d} \xi}=\frac{\mathrm{d} \Phi}{\mathrm{d} \xi}=0 \quad$ as $\quad \xi \rightarrow \infty$

as corresponds to matching with the chemically frozen convectivediffusion solution that exists on the fuel side of the reaction layer.

The boundary-value problem defined in (53)-(56) can be integrated numerically with $L_{\mathrm{H}_{2} \mathrm{O}_{2}}=1.1$ and $L=0.3$ for different values of the parameter $\tau>0$. As can be seen in the sample solutions shown in Fig. 8, the resulting profiles of $\theta$ and $\Phi$ increase monotonically from the zero values achieved at the air-side boundary $\xi=0$ to reach maximum values $\theta_{\max }$ and $\Phi_{\max }$ as $\xi \rightarrow \infty$. It is found that, for any given value of $\tau>0$ smaller than a critical value $\tau_{i}=0.329$, there exist two different solutions, and no solution exists for $\tau>\tau_{i}$, giving a result like that found with one-step Arrhenius chemistry [5]. This can be seen clearly from the results plotted in Fig. 9.

The critical value $\tau_{i}=0.329$, corresponding to the turning point in the curves of Fig. 9, identifies ignition conditions. This value can be used together with (44), (50), and (52) to determine, for a given value of $\theta_{-\infty} \gg 1$, the corresponding ignition time $t_{i}$. The result of the evaluation is seen to be largely independent of values of the specific pair of constants $(A, B)$, selected from Fig. 5, for a given value of $T_{-\infty}$; differences are always smaller than $10 \%$, so that the value of $t_{i}$ effectively is a function only of $\theta_{-\infty}$. The prediction obtained with $A=1 /(2 \mathcal{M})$ and $B=4.01$ used in evaluating (44) and (50) is compared in Fig. 7 with the results of the numerical

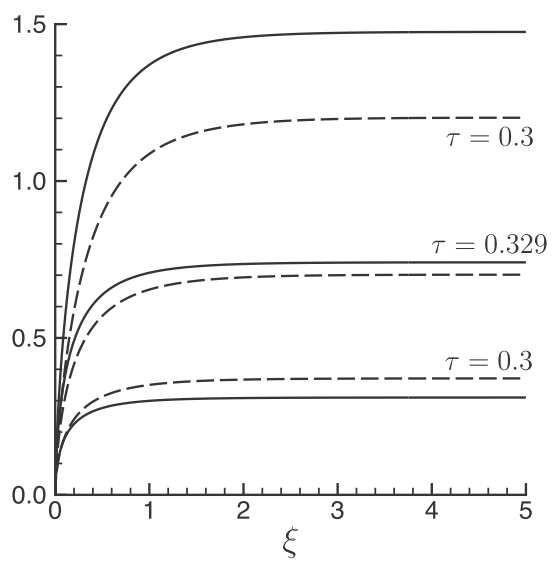

Fig. 8. Profiles of $\theta$ (solid curves) and $\Phi$ (dashed curves) obtained from the solution of (53)-(56) for $\tau=0.3$ and $\tau=0.329$.

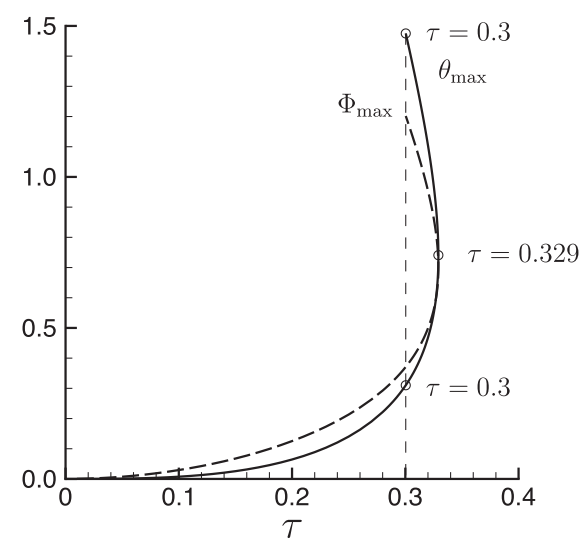

Fig. 9. The variation with $\tau$ of the maximum values $\theta_{\max }$ and $\Phi_{\max }$ obtained as $\xi \rightarrow \infty$ from the solution of (53)-(56).

integrations of (39) and (40). In this figure, as a result of the relatively large errors of order $\eta_{R}^{-2} \sim\left(4 L \ln \theta_{-\infty}\right)^{-1}$ introduced in the asymptotic analysis by neglecting the accumulation terms in writing the problem *(53)-(56), the asymptotic prediction, although approaching the numerical results as $\theta_{-\infty}$ increases, still shows significant departures for values of $\theta_{-\infty}$ beyond the maximum value $\theta_{-\infty} \sim 25$ expected in typical applications. Corrections to the above asymptotic prediction could be obtained in principle by extending the analysis of the limit $\theta_{-\infty} \gg 1$ to a higher order, including in particular unsteady effects that were neglected at leading order, but such an analysis is not further pursued here.

\section{Concluding remarks}

We have seen how the ignition of hydrogen-air diffusion flames in unstrained mixing layers can be investigated by combining a previously derived two-step reduced mechanism with asymptotic methods based on the large activation energies of the controlling reaction-rate constants, yielding a description that in many respects resembles that previously encountered in classical analyses of mixing-layer ignition. The development leads to simplified results for ignition times that provide reasonably good accuracy under most conditions in systems involving only temperatures below crossover, but that do require numerical solution of a two-equation parabolic problem. On the other hand, an explicit ignition-time formula is obtained for hot-air ignition pertaining to the situation typically encountered in gas-turbine applications. Unfortunately, however, for this problem the leading-order result includes logarithmic errors that are much too large, producing underpredictions of ignition times by about an order of magnitude. Future work should investigate this limiting case by considering higher-order corrections in the thermal-runaway analysis.

Until such future work is performed, use of the solid curves in Fig. 7, along with Eqs. (34), (36), and (38), may be recommended for ignition-time estimates. Although strictly they do not apply for hot air-ignition, because of the quasi-isothermal simplifications adopted in their derivation, they nevertheless yield ignition-time values within about $20 \%$ of the correct values even in that limit, as inferred from the comparisons in Fig. 3. It is thus noteworthy that, over the full range of conditions below crossover, suitably normalized non-dimensional ignition times in hydrogen-air mixing layers essentially depend on only two non-dimensional parameters, one measuring the temperature difference between the two streams and the other defining the distance from crossover in terms of the ratio of two reaction-rate constants. 


\section{Acknowledgments}

This work was supported by the US AFOSR Grant \# FA9550-121-0138, by the Comunidad de Madrid through Project \# P2009/ ENE-1597, and by the Spanish MCINN through Project \# CSD2010-00011.

\section{Appendix A. Diffusion of minor species in a binary mixture}

In non-premixed hydrogen-air ignition processes the minor species such as radicals and other reaction intermediates present in low concentrations diffuse into a mixture whose main components are hydrogen, nitrogen, and oxygen. Since nitrogen and oxygen are very similar, they can be treated in the first approximation as a single species. Neglecting the presence of minor species in computing the diffusion velocities of hydrogen and air, $\mathbf{V}_{\mathrm{H}_{2}}$ and $\mathbf{V}_{\mathrm{A}}$, then yields [11]

$Y \mathbf{V}_{\mathrm{H}_{2}}=-(1-Y) \mathbf{V}_{\mathrm{A}}=-D_{\mathrm{H}_{2}-\mathrm{A}}^{\prime}(\nabla Y-0.29 Y \nabla T / T)$

when solving the multicomponent diffusion equation with barodiffusion neglected and with a constant value -0.29 assumed for the Soret factor [12]. Here, $D_{\mathrm{H}_{2}-\mathrm{A}}^{\prime}$ denotes the binary diffusion coefficient of $\mathrm{H}_{2}$ and air.

While thermal diffusion is retained in deriving (A-1), its effect on the diffusion velocity of most minor species, including $\mathrm{H}_{2} \mathrm{O}_{2}$, can be neglected in the first approximation, so that the solution for the corresponding diffusion velocity $\mathbf{V}_{i}$ follows from the Stefan-Maxwell equation written in the form

$\nabla X_{i}=X_{i}\left(\frac{X\left(\mathbf{V}_{\mathrm{H}_{2}}-\mathbf{V}_{i}\right)}{D_{i-\mathrm{H}_{2}}^{\prime}}+\frac{(1-X)\left(\mathbf{V}_{\mathrm{A}}-\mathbf{V}_{i}\right)}{D_{i-\mathrm{A}}^{\prime}}\right)$

in terms of the diffusion velocities $\mathbf{V}_{\mathrm{H}_{2}}$ and $\mathbf{V}_{\mathrm{A}}$ and the binary diffusion coefficients of species $i$ into $\mathrm{H}_{2}$ and air, $D_{i-\mathrm{H}_{2}}^{\prime}$ and $D_{i-\mathrm{A}}^{\prime}$. Here, $X_{i}$ $(\ll 1)$ and $X$ represent the mole fractions of minor species and hydrogen, respectively, which can be expressed in terms of their corresponding mass fractions $Y_{i} \ll 1$ and $Y$ in the form

$X=\frac{\mathcal{M} Y}{1+(\mathcal{M}-1) Y} \quad$ and $\quad X_{i}=\frac{\mathcal{M}_{i} Y_{i}}{1+(\mathcal{M}-1) Y}$

with $\mathcal{M}_{i}$ denoting the air-to-minor-species molecular-mass ratio. Using (A-1) together with (A-3) reduces $(A-2)$ to

$$
\begin{aligned}
\mathbf{V}_{i}\left(\frac{\mathcal{M} Y}{D_{i-\mathrm{H}_{2}}^{\prime}}+\frac{(1-Y)}{D_{i-\mathrm{A}}^{\prime}}\right)= & -[1+(\mathcal{M}-1) Y] \frac{\nabla Y_{i}}{Y_{i}}+(\mathcal{M}-1) \nabla Y \\
& +\left(\frac{\mathcal{M}}{D_{i-\mathrm{H}_{2}}^{\prime}}-\frac{1}{D_{i-\mathrm{A}}^{\prime}}\right) Y \mathbf{V}_{\mathrm{H}_{2}}
\end{aligned}
$$

which can be used to determine explicitly the diffusion velocity $\mathbf{V}_{i}$. For species such that the relative contribution from the terms in the second line in (A-4) is also relatively small, the resulting equation simplifies to give the Fickian law

$Y_{i} \mathbf{V}_{i}=-D_{i}^{\prime} \nabla Y_{i}$

in terms of the composition-dependent diffusivity coefficient $D_{i}^{\prime}$ such that

$\frac{D_{i}^{\prime}}{D_{i-\mathrm{A}}^{\prime}}=\frac{1+(\mathcal{M}-1) Y}{1+\left(\mathcal{M} D_{i-\mathrm{A}}^{\prime} / D_{i-\mathrm{H}_{2}}^{\prime}-1\right) Y}$,

which appropriately reproduces the limiting values $D_{i}^{\prime}=D_{i-\mathrm{H}_{2}}^{\prime}$ and $D_{i}^{\prime}=D_{i-\mathrm{A}}^{\prime}$ for $1-Y \ll 1$ and $Y \ll 1$, respectively. In particular, the simplified law (A-5) is seen to provide a good description for $\mathrm{H}_{2} \mathrm{O}_{2}$ diffusion across the mixing layer, and will be correspondingly used in writing (29).

\section{References}

[1] B. Lewis, G. von Elbe, Combustion, Flames, and Explosions of Gases, second ed., Academic Press, New York, 1961.

[2] C. Treviño, Ignition phenomena in $\mathrm{H}_{2}-\mathrm{O}_{2}$ mixtures, Prog. Astronaut. Aeronaut. 131 (1991) 19-43.

[3] P. Boivin, A.L. Sánchez, F.A. Williams, Combust. Flame 159 (2012) 748-752.

[4] G. del Álamo, F.A. Williams, A.L. Sánchez, Combust. Sci. Technol. 176 (2004) $1599-1626$.

[5] A. Liñán, A. Crespo, Combust. Sci. Technol. 14 (1976) 95-117.

[6] R.J. Kee, G. Dixon-Lewis, J. Warnatz, M.E. Coltrin, J.A. Miller. A Fortran computer code package for the evaluation of gas-phase, multicomponent transport properties, Sandia National Laboratories, Albuquerque, NM, 1986, Report SAND 86-8246.

[7] P. Saxena, F.A. Williams, Combust. Flame 145 (2006) 316-323. <http:// maemail.ucsd.edu/combustion/cermech>.

[8] P. Boivin, A. Dauptain, C. Jimenez, B. Cuenot, Combust. Flame 159 (2012) 1779_ 1790.

[9] M.D. Smooke, Proc. Combust. Inst. 34 (2013) 65-98

[10] D.E. Rosner, Transport Processes in Chemically Reacting Flows, Dover, 2000.

[11] F.A. Williams, Combustion Theory, second ed., Benjamin Cummings, Menlo Park, CA, 1985. p. 634.

[12] M. Fristrom, L. Monchick, Combust. Flame 71 (1988) 89-99.

[13] A.L. Sánchez, M. Vera, A. Liñán, Phys. Fluids 18 (2006) 078102.

[14] A.L. Sánchez, E. Fernández-Tarrazo, P. Boivin, A. Liñán, F.A. Williams, C.R. Mech. 340 (2012) 882-893. 